\title{
Harmonic Analysis and Dynamical Response Optimization in Ceramic Tile Finishing
}

\author{
A. Pavlovic ${ }^{1, *}$, G. Minak ${ }^{1}$, G. Lucisano ${ }^{2}$, F. Sorcinelli ${ }^{1}$ \\ ${ }^{1}$ Alma Mater Studiorum University of Bologna, Interdepartmental Industrial Research Centre of \\ Advanced Applications in Mechanical Engineering and Materials Technology, Viale \\ Risorgimento 2, 40136 Bologna, Italy \\ e-mail: ana.pavlovic@unibo.it \\ e-mail: giangiacomo.minak@unibo.it \\ e-mail: sorcinelli.f@gmail.com \\ ${ }^{2}$ Alma Mater Studiorum University of Bologna, Interdepartmental Industrial Research Centre for \\ Buildings and Constructions, Viale Risorgimento 2, 40136 Bologna, Italy \\ e-mail: giuseppelucisano@gmail.com \\ *corresponding author
}

\begin{abstract}
This paper investigates the dynamic response of a large production machine used for surface finishing of oversized ceramic tiles through tool machining. In particular, the study aims at improving this phase of the industrial process, as well as the overall quality of the final products. As the finite element method has become an essential solution technique in many areas of engineering related to dynamic processes, the FEM is utilized in the optimization of machine basement for ceramic tile finishing. Two different basements of cutting machine have been considered as alternative design solutions. The responses in frequency and phase have been observed and compared. Stresses and strains have been also considered for comparison. This numerical computation permitted to select a convenient design option for the basement.
\end{abstract}

Keywords: Ceramic manufacturing, process quality, machine design, harmonic analysis, vibration, finite element, ANSYS.

\section{Introduction}

Most human activities involve vibrations in one form or another. For example, we hear thanks to our timpani vibrating and see thanks to the light waves undergoing vibrations. Breathing is associated with the vibration of the lungs and the ability to walk depends on the oscillatory motion of the legs and hands. The term "vibration" refers to an oscillation of a mechanical system around a point of balance. A typical example of a forced vibration is a car that runs through a disassembled road or a washing machine centrifuge. The first researchers in the field of vibration mainly concentrated their efforts on understanding natural phenomena and the development of mathematical theories able to describe the vibration of physical systems. A general review on the dynamics of mass variable systems is proposed in Cveticanin (2012) while Cetkovic and Vuksanovic (2012), detailing the influence of boundary conditions on vibration of plates, shows a practical example about the complexity embraced by vibration phenomena. 
More recently, a high number of investigations have been related to the effect of vibrations in the case of engineering applications, such as machinery design, foundations, structures, engines, turbines, condition monitoring and diagnostics of machines and mechanical systems in general. Vibrations are in fact present almost everywhere in industry and engineering (Weiss and Ricci 1981, Singiresu, 2004). Indeed, alternative motors show vibrations during their operation due to the rotor imbalance (possibly related to design defects or poor manufacturing quality), thus acting as sources of vibrations, which affect the whole structure used for their support. In addition, high inertia actions and transient loads associated with operation at high speed can induce vibrations as well (Altintas and Weck 2004, Troncossi et al. 2009, Martini et al. 2016).

In these quite common situations, structures and frames may fail due to the fatigue of the material resulting from the cyclic variation of loads even if in the case of relatively low levels of loads. Moreover, vibrations can cause rapid wear of different parts of machinery, such as bearings and gears, creating excessive noise or additional gaps and vibrations. In particular, in tool processing, as in the case under investigation, vibrations can lead to wrong dimensions of the final product, poor surface finishing, and even cracks in the cutting tools or heads (Altintas 2012).

When the natural frequency of a machine or structure falls within the range of the frequency content of one of these external excitations, the corresponding vibration mode can be excited, leading to excessive deformation and breakage. Furthermore, in the case of impulsive loads a wide frequency range can be excited (Martini et al. 2013 and 2014). Technical literature is full of systems failure reports caused by resonance excitation and excessive vibration of components and systems (Alexious and Wright 1985). Due to the detrimental effects that vibrations may have on machines and structures, vibration analysis has become a standard procedure in the design and development of most engineering systems (Mießen et al. 1976, Fragassa 2015) to ensure their endurance, maximize their efficiency and provide safety.

One of the main purposes of these studies is to reduce the vibration through a proper design of the machines and, more commonly, their supports and connections. Within this context, mechanical engineering attempts to design mechanisms and actuators to minimize transient loads and unbalanced excitations while structural engineering tries to design the supporting structure to ensure that the effect of the imbalance is not harmful. A numerical approach can be easily considered as a practical way to investigate these complex phenomena.

Although the Finite Element Method (FEM) had not been defined yet in the form we know nowadays, the concept of discretization of domain has been already developed by engineers for centuries as an important design resource. For example, ancient mathematicians discretized the circumference of a circle by a polygon characterized by very high number of sides, where each side is almost equivalent to a finite element, nowadays. The finite element method as now known was presented for the first time by Turner Clough, Martin, and Topp in connection with the analysis of aeronautical and aerospace structures, opening the way for a massive application of computation mechanics to engineering, including the vibration studies.

Until the middle of the last century, vibration problems, including those dealing with complex engineering systems, were investigated by numerical methods almost exclusively considering large models and very few degrees of freedom (Marten 1962, Peters et al. 1976).

Then, the advent of high-performance computers, available for the first time in ' 50 , has allowed to progressively handle more complex systems (Mackerle 1999 and 2003). In particular, they have permitted to generate approximate solutions in a semi-defined way, based on classic methods and formula, but also considering a numeric evaluation in the case of some terms that cannot be expressed in closed form. 
This simultaneous improvement, both in terms of performances and discretized models, definitely launched the use of numerical computation in the analysis of dynamics in engineering applications, including manufacturing processes (as summarized in Mourtzis et al. (2014)).

Interesting experiences in the utilization of Finite Elements (FEs) for a better comprehension of the tool cutting processes are largely known, especially in the case of traditional metal cutting. For instance, in Mahdavinejad (2005) the workpiece instability in metal tuning was investigated; in Mahnama et al. (2010 and 2012) the effect of chip formation was taken into account inside the simulation models. Furthermore, FEs can also be utilized for the optimization of production plants, as in Myers et al. (2003) where the structural dynamics of a whole vertical milling machine was improved, or some specific parts as in Assefa (2013) and Okubo and Yoshida (1982), where a modal analysis was focused on the machine tool column. FE methods also permit to analyze complex and uncommon situations as in Kehl and Blank (2015) where the effect on machine vibrations of specific solutions in the drive controls was estimated; or in Cakair and Yahya (2005) where hard turning operations were studied with special attention to the cutting tools prior to the emerging of fracture conditions.

\section{Analysis of vibrations in brief}

When a structure is moving or undergoing large displacements, dynamic effects have to be taken seriously. Various forms of analyses can be used for this purpose. Particularly, a harmonic analysis was chosen for the present investigation.

Harmonic analysis (Stain and Marphy 1993) is the branch of mathematical analysis that studies the representation of functions or signals as overlapping of fundamental waves. It is based on the concepts of the Fourier series and transform, which are exploited to extend the analysis to different areas. The fundamental waves are called 'harmonics', hence the name 'harmonic analysis'.

In practice, a harmonic analysis studies the behavior of a complex physical system by solving linear differential equations through numerical methods. As a final result, one can deduce the response of a physical system to different excitations in a frequency domain by defining its harmonic response.

The harmonic response function represents a demonstration of stationary linear systems closely related to the transfer function and therefore equivalent to differential equations (with systems initially in silence). Often, however, this different formulation proved to be more advantageous due to some of its main features, including the possibility of being experimentally detected. It follows that the harmonic response function represents, with respect to the differential equation describing the problem, a mathematical model of easier identification from experimental data.

As input, to be correctly performed, a harmonic analysis needs harmonic loads (such as forces, pressures or imposed displacements) of known magnitude and frequency (Eman and Kim 1983). Beyond the peculiarity of these multiple loads, they have to be applied at a specific and constant frequency. Forces and displacements can be in-phase or out-of phase. As output, a harmonic analysis provides harmonic displacements at each degree of freedom (DOF), usually out of phase with the applied loads, but also other derived and useful quantities, such as stresses and strains (Herrin 2012, Liang et al. 2013). Thanks to the information it provides, the harmonic analysis can be used to make sure that a given design can withstand sinusoidal loads at different frequencies (e.g., an engine running at different speeds), but also to detect resonant responses and avoid them if necessary (by using dampers, for example). 
In the present case, a harmonic analysis has been used to analyze the dynamic response of a machine tool comparing different design solutions for a wide frequency range of loads (Tlusty and Moriwaki 1976, Van der Auweraer 2001).

The following equations show the correlation between an imposed displacement and the resulting acceleration for harmonically excited structures. The harmonic motion of a point as a function of frequency can be written as:

$$
x(\omega t)=A \sin (\omega t)
$$

where

$\mathrm{x}=$ displacement

$\mathrm{A}=$ amplitude of excitation

$\omega=$ frequency of excitation

$\mathrm{t}=$ time

Velocity and acceleration may be written as:

$$
\begin{gathered}
\dot{x}(\omega t)=\omega A \cos (\omega t) \\
\ddot{x}(\omega t)=-\omega^{2} A \sin (\omega t)
\end{gathered}
$$

Looking at peak response and considering $A=x$ because of the non-zero displacement constraint:

$$
\ddot{x}=-\omega^{2} x \quad \ddot{x}=\left|\omega^{2} x\right|
$$

\section{Materials and Methods}

The research aims, in fact, at improving the dynamic behavior of a wide machine used for (super) finishing porcelain stoneware tiles by changes in the structural design. The machine, represented in Fig. 1, uses a tool cutting process for polishing and then lapping the ceramics. The surface treatment is realized by a swinging motion on the ceramic tiles of the heads containing the cutting tools. This movement is operated by two heavy beams, transversally oscillating. Speeds are controlled in order to meet every kind of manufacturing requirement (Pavlovic and Fragassa 2016, Fragassa 2016, Fragassa 2016).

In terms of structural parts, this machine consists in a basement, a left beam (for polishing), a central bridge, a right beam (for lapping) and several motors with tool heads. The tool heads (sixteen) exercise a force on the tiles thanks to pneumatic pistons. The movement of these heavy beams follow a sinusoidal law and represents the main source of vibration. All these parts are realized in structural steel $\left(E=210 \mathrm{GPa}, v=0.3, \rho=7860 \mathrm{~kg} / \mathrm{m}^{3}\right)$. 

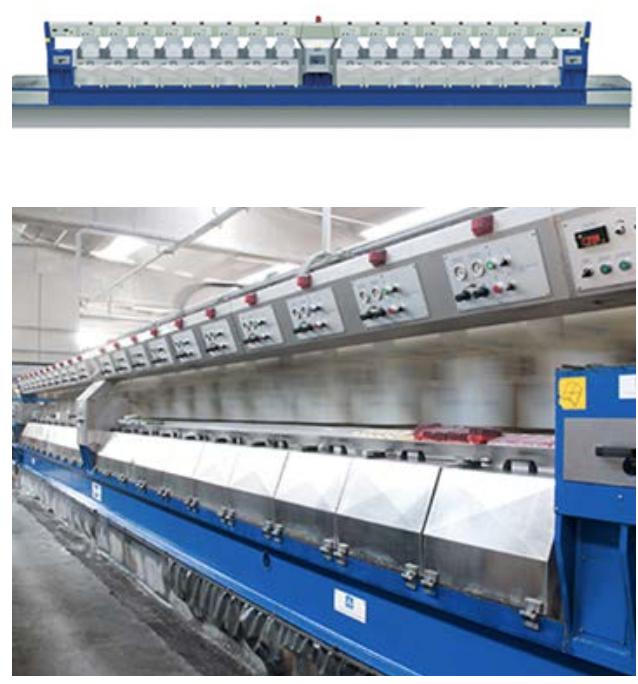

Fig. 1. Machine for tile surface finishing (polishing and lapping)

In our case, this harmonic study was anticipated by a modal analysis by the same authors, presented in Pavlovic et al. (2016), which represents the necessary premise. Modal analysis is a technique to study the dynamic characteristics of a structure under vibrational excitation. Natural frequencies, mode shapes and mode vectors of a structure can be determined using modal analysis. It allows to avoid resonant vibrations or to vibrate at a specified frequency and gives engineers an idea of how the design will respond to different types of dynamic loads. In this previous investigation, not only preliminary results on the dynamical behaviour of the machine (as its modal frequencies, etc.) are available, but also useful information regarding the strategy to simplify the physical system (geometry, forces, constrains) in the way to perform a convenient numerical analysis.

In brief, the main difference between the two studies is the fact that modal analysis does not consider the structure response under a dynamic load, but only provides information on the system natural frequencies, which solely depend on mass distribution, geometries and constraints. Modal analysis is a type of analysis that usually represents the first step to be performed before a more complex dynamic analysis, such as harmonic analysis, which points to the structure verification under dynamic condition of loads. In a structure, as said, a constant cyclic load will produce a constant or harmonic cyclic response. The results of the harmonic analysis are used to determine the stationary response of a linear structure to loads that are sinusoidal (harmonically) over time. It is thus possible to ascertain whether the structures will or will not be able to overcome the effects of resonance, fatigue and other harmful effects related to external vibrations. It is also noteworthy that this analysis technique regarding harmonic forces only calculates the stationary vibrations present on a structure. Transient vibrations, which occur in response to the initial stimulus, are not considered in this first harmonic analysis. 

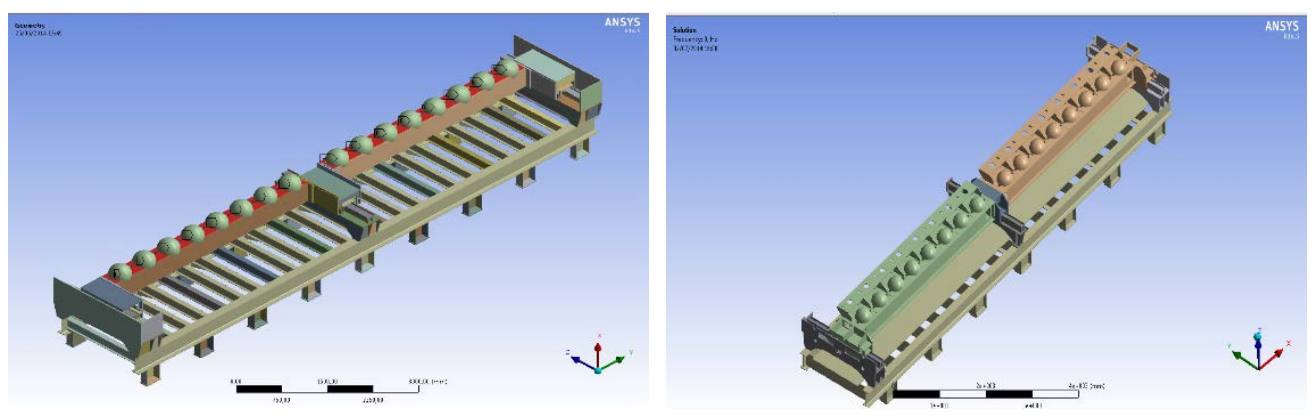

Fig. 2. Original geometry and advanced geometry for the frame

The initial geometry was imported from Inventor in .stp format. Engines were simplified using concentrated masses of $510 \mathrm{~kg}$ each and force with magnitude of $24 \mathrm{kN}$. The machine presented an overall weight of 15,300 kg (Fig. 2a). After other geometrical simplifications, the Finite Element discretization was created and the constraints set. Also, 1,421,069 nodes and 624,873 elements of $30 \mathrm{~mm}$ size were used (Fig. 3a).

The advanced geometry was similarly imported and simplified (Fig. 2b). In this case, each motor presented a mass of $307 \mathrm{~kg}$ for an overall machine weight of 10,578 $\mathrm{kg}$. The final mesh contained 812,449 nodes and 408,614 elements of $30 \mathrm{~mm}$ in size (Fig. 3b).
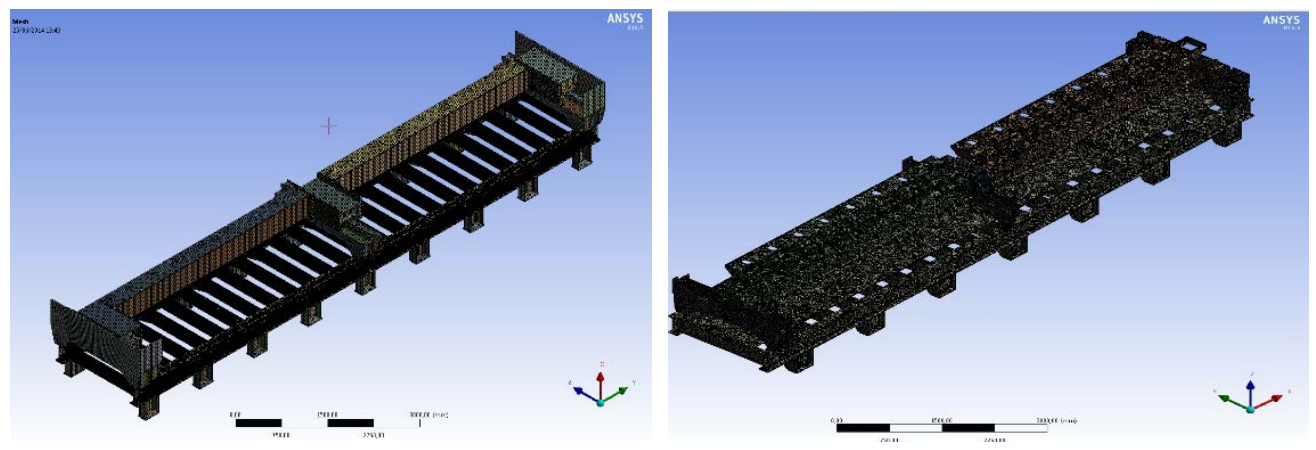

Fig. 3. Mesh in the case of original geometry and advanced geometry

It is noteworthy that the main differences between the original and advanced geometries were not limited in weight and thinness, but also the design solutions used for connecting the heavy beams (with motors) to the basement were involved (details in Fig. 4). The analysis has been realized considering an external harmonic load varying on a range of frequency between 0 $160 \mathrm{~Hz}$. Ten modes have been considered (Fig. 5). 

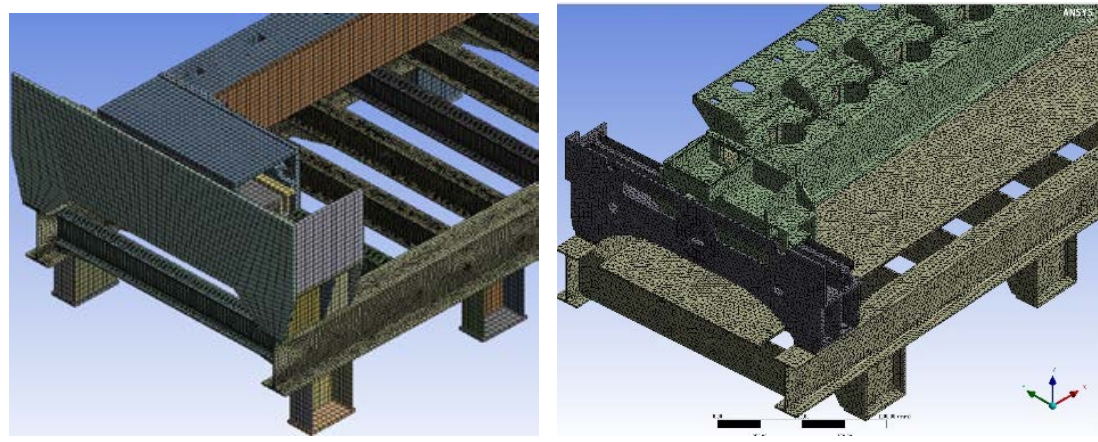

Fig. 4. Details of mesh in the case of original and advanced geometries

\section{Results and Discussion}

Frequency-response analysis considers loading at one frequency only. Loads may be out-ofphase with one another, but the excitation is at a known frequency. In a harmonic analysis, required inputs are Young's Modulus, Poisson's Ratio, and Mass Density while all other material properties can be specified, but are dispensable.

It is useful to note at this point that ANSYS WB used to perform this study, does not support Full solution method, so it does not support a given displacement support in a harmonic analysis. Not all available loads support phase input. Accelerations, bearing load, and moment load will have a phase angle of $0^{\circ}$. If other loads are present, shift the phase angle of other loads, such that the acceleration, bearing, and moment loads will remain at a phase angle of $0^{\circ}$.

Since modal coordinates are used, a harmonic solution using software ANSYS WB use the Mode Superposition method. Previous modal analysis automatically determines the number of modes ' $n$ ' necessary for an accurate solution. Although a free vibration analysis is performed first, the harmonic analysis portion is very quick and efficient. Mode Superposition method is usually much faster overall than the Full method. Initial free vibration analysis is performed so that harmonic simulations will know what the natural frequencies of the structure are. In a harmonic analysis, the peak response will correspond with the natural frequencies of the structure. Nonzero prescribed displacements are not possible because the solution is done with modal coordinates. Simulation can cluster the results near the natural frequencies instead of using evenly space results. The cluster number determines how many results on either side of a natural frequency is solved. Frequency and phase responses were analyzed for the studied geometry.

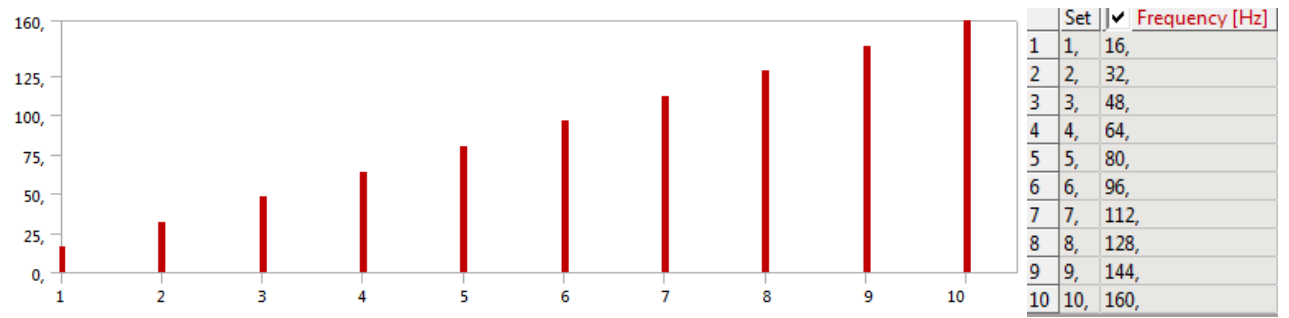

Fig. 5. Harmonic frequency of the ten initial modes

Three types of results are available: 
Contour results of components of stresses, strains, or displacements for surfaces, parts, and/or assemblies at a specified frequency and phase angle

Frequency response plots of minimum, maximum, or average components of stresses, strains, displacements, or acceleration at selected vertices, edges, or surfaces.

Phase response plots of minimum, maximum, or average components of stresses, strains, or displacements at a specified frequency.

Machines for tile finishing should not vibrate, but some period of vibrations use to occur because of self-weight and other operations that are performed on it. Contour results for both basement solutions, are available for stress and total displacement at a given frequency and phase angle (Figs. 6-9).
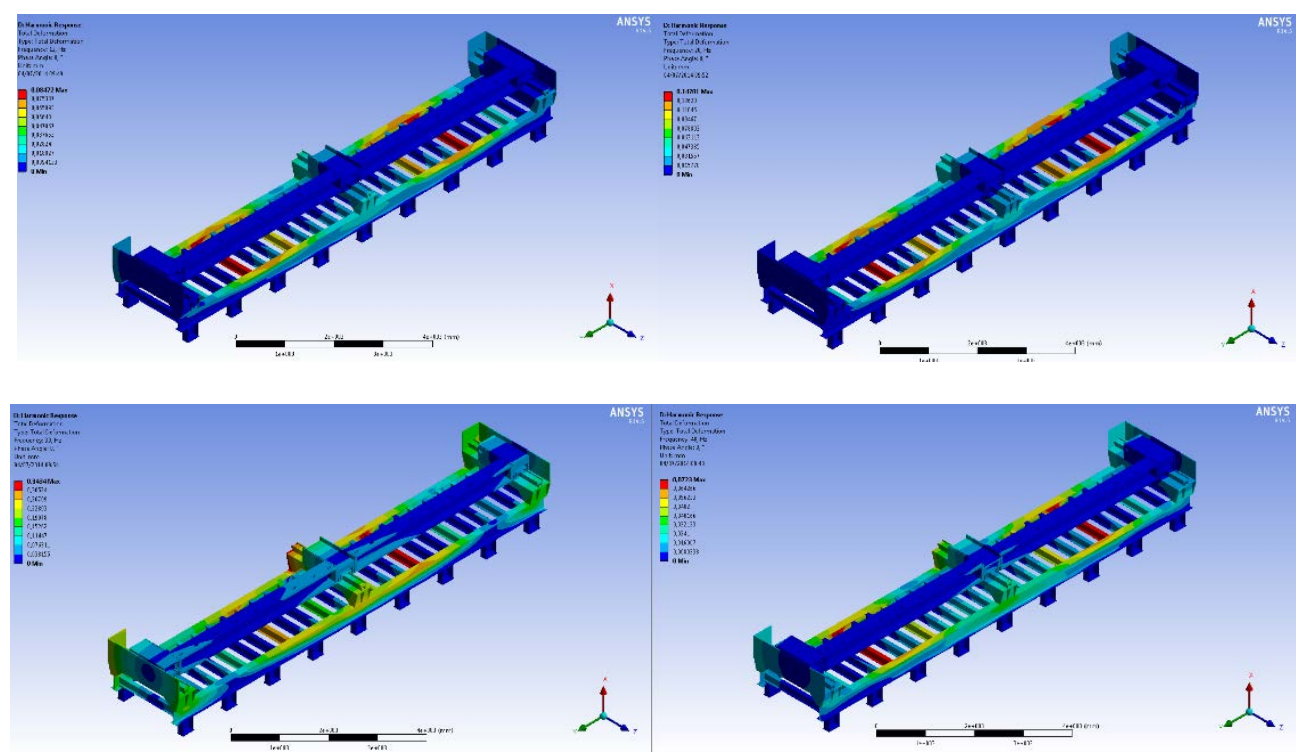

Fig. 6. Harmonic response in terms of total deformations in the case of the original solution

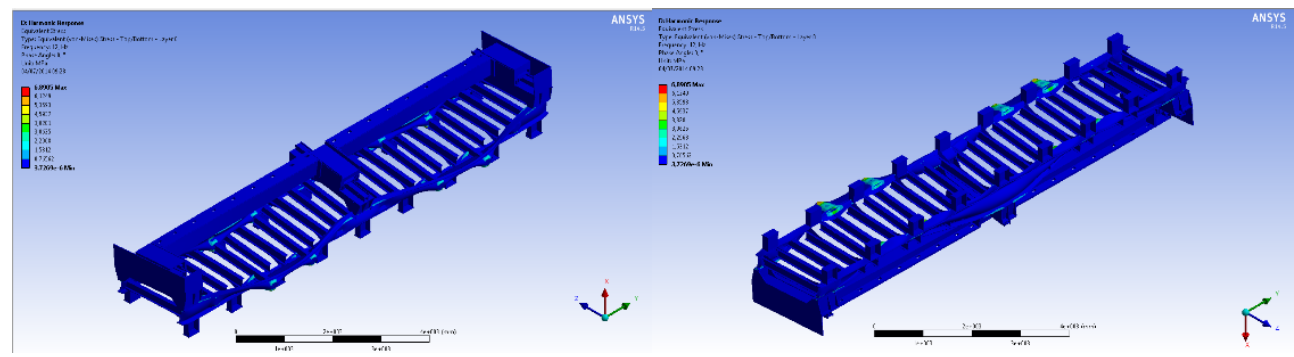

Fig. 7. Harmonic response in terms of equivalent stress in the case of the original solution (top and bottom view) 

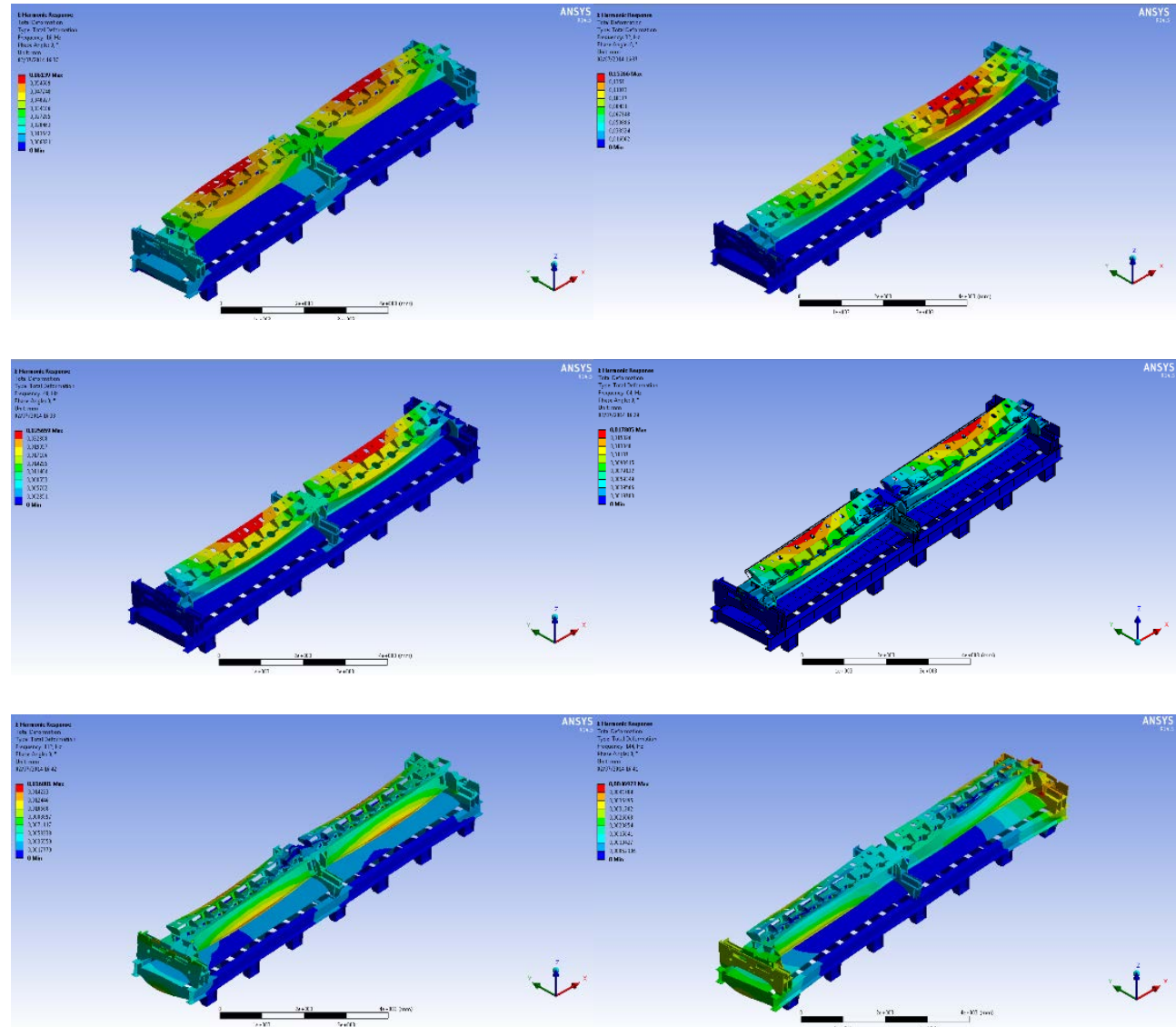

Fig. 8. Harmonic response in terms of total deformations in the case of the advanced solution

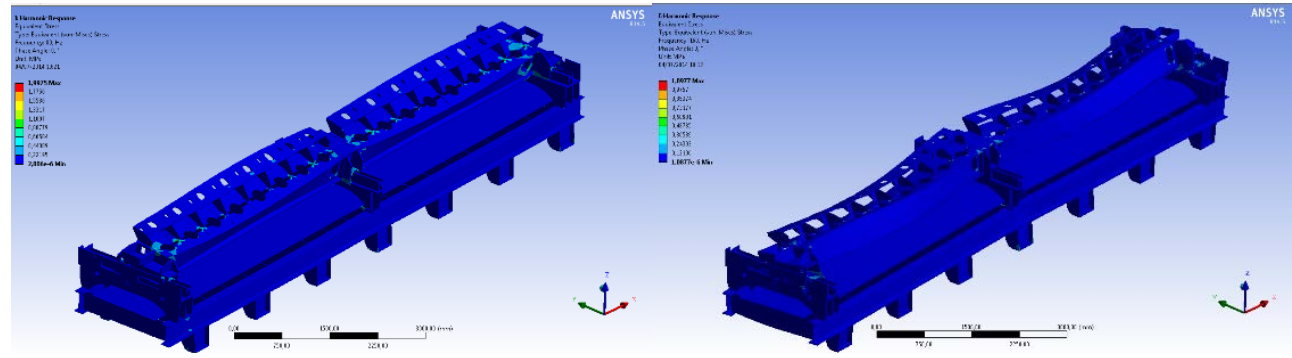

Fig. 9. Harmonic response in terms of equivalent stress in the case of the advanced solution

The Full method is an alternate way of solving harmonic analyses. In the Full method, matrix equation is solved directly in nodal coordinates, analogous to a linear static analysis except that complex numbers are used, as shown in Eq. (5):

$$
\left[\mathrm{K}_{\mathrm{C}}\right]\left\{\mathrm{X}_{\mathrm{C}}\right\}=\left\{\mathrm{F}_{\mathrm{C}}\right\}
$$

In the Mode Superposition method, a simpler set of uncoupled equations is solved for. In the Full method, a more complex, coupled matrix [KC] must be factorized. Because of this, the Full method tends to be more computationally expensive than the used Mode Superposition method. ANSYS Workbench can perform a harmonic analysis on a structure, determining the steady-state 
sinusoidal response to sinusoidal varying loads all acting at a specified frequency. When the input acceleration is a function of frequency, as seen above, the Frequency Response where the load is applied can be traced in the Solution output as in Fig. 10.

a)

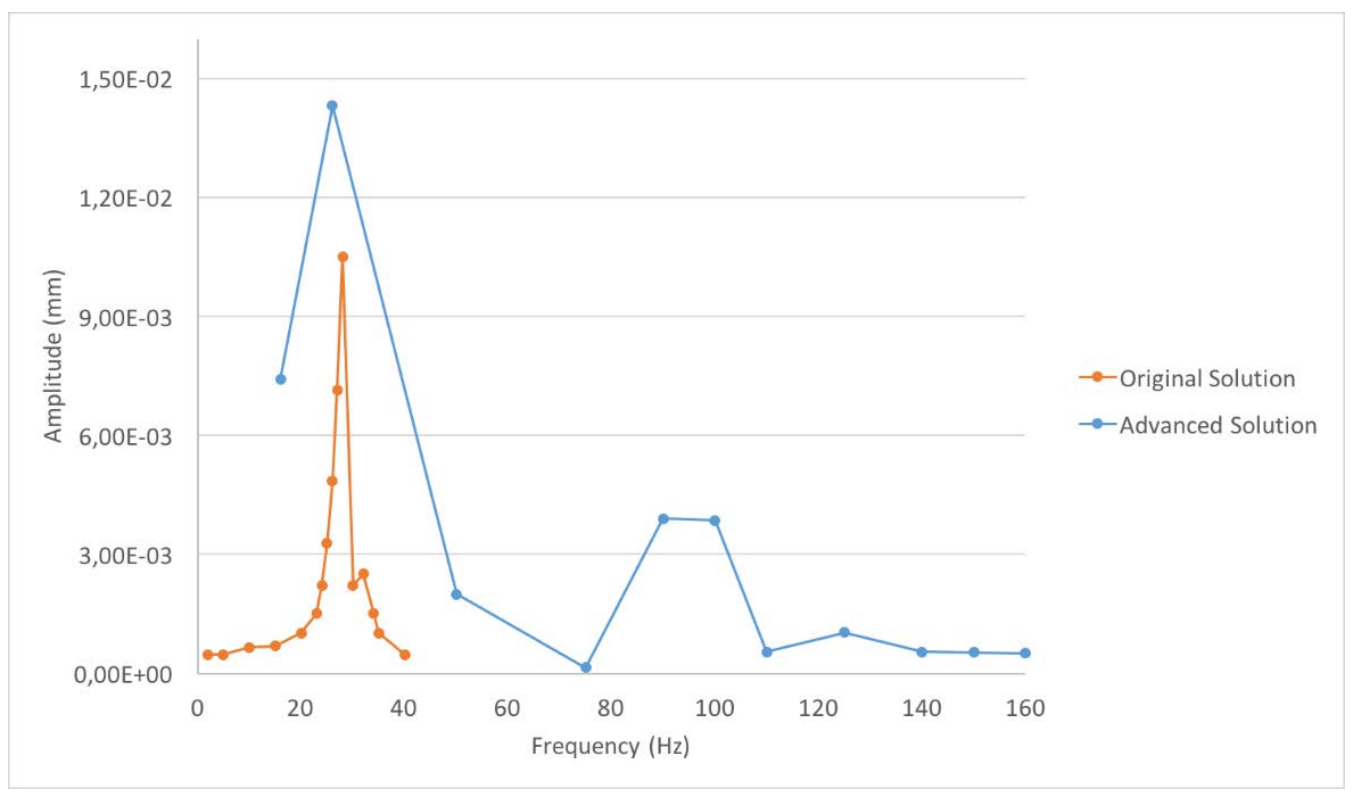

b)

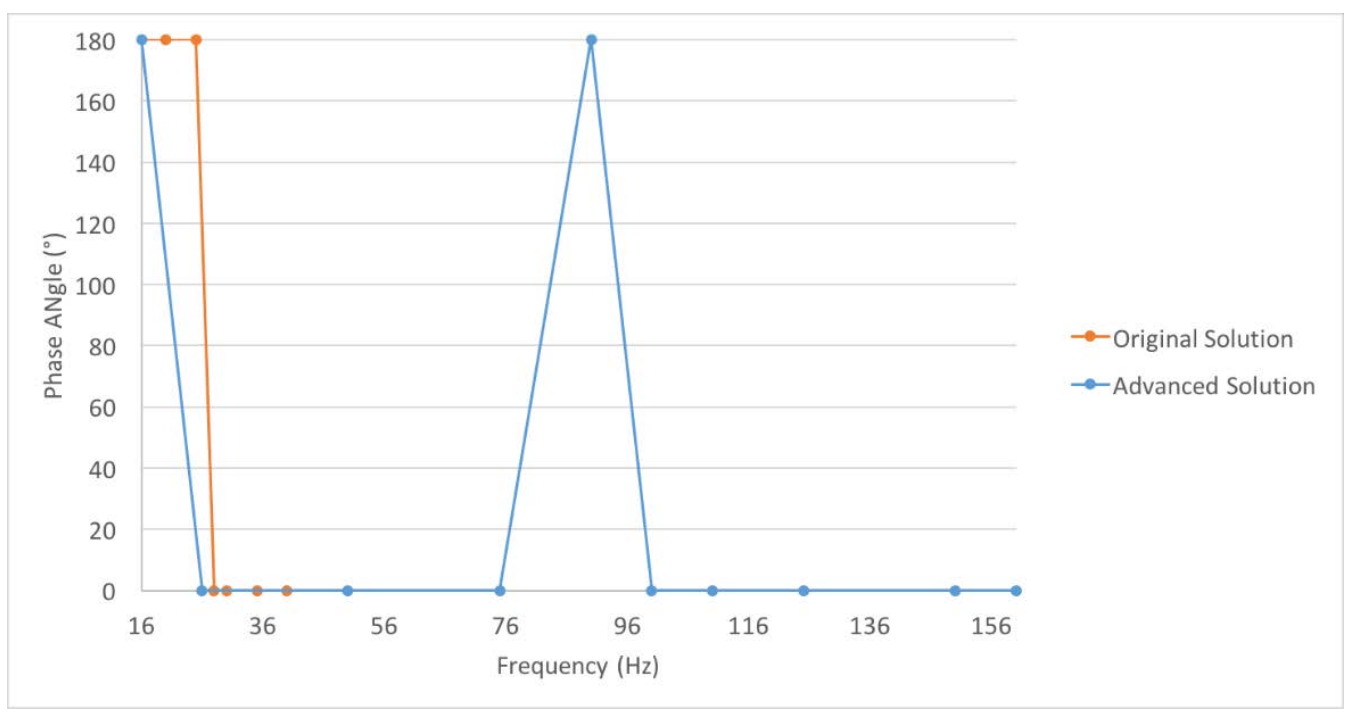

Fig. 10. Comparing a) Amplitude and b) Phase angle for original and advanced solution

In this case, two design solutions were compared in terms of frequencies and modes, permitting to validate and optimize the structures in terms of vibrations. In particular, it was noted that, while the original design presents a larger gamma of geometries for deformed 
configurations, mainly involving the basement and its parts, the improved design focuses these deformed configurations on beams, which are fixed on an extremely stable basement. This second case presents several advantages with the aim at providing the highest quality in process or product.

\section{Conclusions}

Finite Element Analysis of the harmonic behavior of the tile finishing machine shows that the numerical analysis provides an estimation of the maximum stress that can be used as an approach to be joint to the experimental procedures. This leads to the conclusion that only the proposed numerical procedure should be used when designing and optimizing machines for tile finishing. The presented analysis approach can help the designer to accurately determine the maximum stress, which can then be used for optimizations of machine for tile finishing.

The finishing machine under investigation serves to grind porcelain stoneware and similar tiles with workable widths from $300 \mathrm{~mm}$ to $1600 \mathrm{~mm}$. The study focused on analyzing the dynamic response of the production plant by a harmonic simulation. Harmonic analysis is a technique to determine the steady state response of a structure to sinusoidal (harmonic) loads of known frequency. This numerical simulation helped to compare the effect of design changes on the machine basement with respect to the expected harmonic loads. In particular, two different basements were considered and compared considering the stresses/strains effects of loads. Furthermore, the computational approach was validated as convenient option with the aim at optimizing specific components and the whole production line.

\section{Acknowledgements}

This research has been realized inside the project IPERCER with the aim at developing energy efficient solutions for the production of large porcelain stoneware tiles. The authors acknowledge support of the European Union and the Region Emilia-Romagna (inside the POR-FESR 20142020, Axis 1, Research and Innovation)

\section{References}

Alexiou K, Wright JR (1985). Determination of force distribution in multi-point resonance testing using various direct methods, 2nd International Symposium on Aero elasticity and Structural Dynamics, 553-564.

Altintas Y, Weck M (2004). Chatter stability of metal cutting and grinding, Annals of the CIRP, 53 (2), 619-642.

Altintas Y (2012). Manufacturing Automation: Metal Cutting Mechanics, Machine Tool Vibrations, and CNC Design, Cambridge University Press.

Assefa M (2013). Modal Analysis of Machine Tool Column Using Finite Element Method, International Journal of Mechanical, Aerospace, Industrial, Mechatronic and Manufacturing Engineering, 7, 4, 699-708.

Cakair CM, Yahya IS (2005). Finite element analysis of cutting tools prior to fracture in hard turning operations, Materials and Design, 26, 2, 105-112.

Cetkovic M, Vuksanovic D (2012). Vibrations of isotropic, orthotropic and laminated composite plates with various boundary conditions. Journal of the Serbian Society of Computational Mechanics, 6, 1, 83-96. 
Cveticanin L (2012). A review on dynamics of mass variable systems. Journal of the Serbian Society of Computational Mechanics, 6, 1, 56-73.

Eman KF, Kim KJ (1983). Modal Analysis of Machine Tool Structures Based on Experimental Data, Journal of Engineering for Industry, 105, 282-287.

Fragassa C (2015). Limits in Application of International Standards to Innovative Ceramic Solutions. International Journal for Quality Research, 9, 2, 279-298

Fragassa C (2016). Flexural Testing Machine as an Off-Line Control System for Quality Monitoring in the Production of Bended Ceramic Tiles. International Journal for Quality Research, 10, 2, 373-388.

Fragassa C (2016). Methodological details in modelling the viscoelastic response of ceramic materials by commercial Finite Elements codes. FME Transactions, 44, 1, 58-64

Herrin DW (2012) Vibro-Acoustic Design in Mechanical Systems, Ansys Tutorial.

Kehl G, Blank D (2015). Finite Element Analysis Simulation of Machine Tools with Integrated Drive Control, Applied Mathematics and Materials, 13-16.

Liang Y, Chen W, Bai Q, Sun Y, Chen G, Zhang Q, Sun Y (2013). Design and dynamic optimization of an ultraprecision diamond flycutting machine tool for large KDP crystal machining, International Journal of Advanced Manufacturing Technology, 69(1), 237-244.

Mackerle J (1999). Finite-element analysis and simulation of machining: a bibliography (19761996), Journal of Materials Processing Technology, 86, 17-44.

Mackerle J (2003). Finite element analysis and simulation of machining: an addendum A bibliography (1996-2002), International Journal of Machine Tools \& Manufacture, 43, 103-114.

Mahdavinejad R (2005). Finite element analysis of machine and workpiece instability in turning, International Journal of Machine Tools \& Manufacture, 45, 753-760.

Mahnama M, Movahhedy MR (2010). Prediction of machining chatter based on FEM simulation of chip formation under dynamic conditions, International Journal of Machine Tools \& Manufacture, 50, 611-620.

Mahnama M, Movahhedy MR (2012). Application of FEM simulation of chip formation to stability analysis in orthogonal cutting process, Journal of Manufacturing Processes, 14, 188-194.

Marten H. R (1962), The use of models for analysis of machine tool structures, International Journal of Machine Tool Design and Research, 2, 153-163.

Martini A, Troncossi M, Rivola A (2013). Elastodynamic effects of mass-balancing: experimental investigation of a four-bar linkage, Advances in Mechanical Engineering, 2013, 1-11.

Martini A, Troncossi M (2016). Upgrade of an automated line for plastic cap manufacture based on experimental vibration analysis, Case Studies in Mechanical Systems and Signal Processing, 3, 28-33.

Martini A, Troncossi M, Carricato M, Rivola A (2014). Elastodynamic behavior of balanced closed-loop mechanisms: numerical analysis of a four-bar linkage, Meccanica, 49(3), 601614.

Mießen MW, Müller W, Prößler EK (1976). Visual Representation of the Dynamic Behaviour of Machine Tool Structures, Annals of the CIRP, 25,1.

Mourtzis D, Doukas M, Bernidaki D (2014). Simulation in Manufacturing: Review and Challenges, Procedia CIRP, 25, $213-22$.

Myers A, Ford DG, Xu Q (2003). Finite element analysis of the structural dynamics of a vertical milling machine, Transactions on Engineering Sciences, 44, 431-440.

Okubo N, Yoshida Y (1982). Application of Modal Analysis to Machine Tool Structures, Annals of the CIRP, 31.

Pavlovic A, Fragassa C (2016). Modelling the Viscoelasticity of Ceramic Tiles by Finite Element. Proceedings of the 8th Conference on Times of Polymers \& Composites. American 
Institute of Physics (AIP). 19-23th June 2016, Ischia (Italy). 1736, 4949749, doi: 10.1063/1.4949749

Pavlovic A, Fragassa C, Ubertini F, Martini A (2016). Modal analysis and stiffness optimization: the case of a tool machine for ceramic tile surface finishing. Journal of the Serbian Society for Computational Mechanics, 10.2, 30-44.

Peters J, Mergeay M (1976). Dynamic Analysis of Machine Tools using Complex Modal Method, Annals of the CIRP, 25, 1.

Singiresu SR (2004). Mechanical Vibrations, Fourth Edition, Pearson Education Inc.

Stain EM, Marphy TS (1993). Harmonic Analysis: Real-Variable Methods, Orthogonality and Oscillatory Integrals, Princeton University Press.

Tlusty J, Moriwaki T (1976). Computational Identification of Dynamic Structural Models, Annals of the CIRP, 25, 2.

Troncossi M, Troiani E, Rivola A (2009). Design optimization of a laser cutting machine by elastodynamic modeling, Proceedings of the 9th Biennial Conference on Engineering Systems Design and Analysis (ESDA), Volume 2, 7-9 July 2008, Haifa, Israel, 551-559.

Van der Auweraer H (2001). Structural Dynamics Modeling using Modal Analysis: Applications, Trends and Challenges, IEEE Instrumentation and Measurement Technology Conference, 3, 1502-1509.

Weiss G, Ricci F (1981). Harmonic Analysis, Proceedings 1981, Springer-Verlag. 